\title{
Inequalities of holographic entanglement of purification from bit threads
}

\author{
Dong-Hui Du ${ }^{1,2, a}$, Fu-Wen Shu ${ }^{1,2,3, b}$, Kai-Xin Zhu ${ }^{1,2, c}$ \\ ${ }^{1}$ Department of Physics, Nanchang University, Nanchang 330031, China \\ ${ }^{2}$ Center for Relativistic Astrophysics and High Energy Physics, Nanchang University, Nanchang 330031, China \\ ${ }^{3}$ Center for Gravitation and Cosmology, Yangzhou University, Yangzhou, China
}

Received: 11 February 2020 / Accepted: 26 July 2020 / Published online: 5 August 2020

(C) The Author(s) 2020

\begin{abstract}
There are increasing evidences that quantum information theory has come to play a fundamental role in quantum gravity especially the holography. In this paper, we show some new potential connections between holography and quantum information theory. Particularly, by utilizing the multiflow description of the holographic entanglement of purification (HEoP) defined in relative homology, we obtain several new inequalities of HEoP under a max multiflow configuration. Each inequality derived for HEoP has a corresponding inequality of the holographic entanglement entropy (HEE). This is further confirmed by geometric analysis. In addition, we conjecture that, based on flow considerations, each property of HEE that can be derived from bit threads may have a corresponding property for HEoP that can be derived from bit threads defined in relative homology.
\end{abstract}

\section{Contents}

1 Introduction ................. 1

2 Review of bit threads . . . . . . . . . . . . 2

2.1 Flow . . . . . . . . . . . . 2

2.2 Threads . . . . . . . . . . . . . 3

2.3 Multiflow . . . . . . . . . . 3

3 Relative homology and generalized HEE . . . . . . 4

4 Properties of HEoP derived from bit threads . . . . . 5

4.1 Multiflow description of HEoP as relative homology ................ 5

4.2 Properties of HEoP derived from bit threads . . 6

4.2.1 Tripartite case . . . . . . . . . . 6

4.2.2 Quadripartite case ... . . . . . 7

5 Entropy cone for HEoP . . . . . . . . . . . . . . . . 8

6 Conclusion ................... 9

a e-mail: donghuiduchn@gmail.com

b e-mail: shufuwen@ncu.edu.cn (corresponding author)

c e-mail: kaixinzhuabc@gmail.com
References . . . . . . . . . . . . . . 9 9

\section{Introduction}

The holographic essence of quantum gravity was elucidated in $[1,2]$, which revealed the duality between the quantum gravity theory in a $(d+1)$-dimensional space-time region and the quantum field theory on the $d$-dimensional boundary of this region. Specifically, in the AdS/CFT correspondence [3-5], the entanglement entropy (the von Neumann entropy) for a spatial region $A$ on conformal boundary was shown to be given by the area of minimal homologous surface [6,7], i.e., the Ryu-Takayanagi (RT) formula

$S(A)=\frac{\operatorname{area}\left(m_{A}\right)}{4 G_{N}}$,

where $m_{A}$ is the minimal surface in the bulk homologous to $A$. This reveals the deep connections between quantum entanglement and space-time geometry.

The entanglement entropy primely characterizes the quantum entanglement in pure bipartite states, and has many known properties. For example,

(1)Araki-Lieb: $|S(A)-S(B)| \leq S(A B)$,

(2) Subadditivity: $S(A B) \leq S(A)+S(B)$,

(3) Strong Subadditivity $1: S(B)+S(A B C)$

$\leq S(A B)+S(B C)$,

(4) Strong Subadditivity $2: S(A)+S(C)$

$$
\leq S(A B)+S(B C) \text {. }
$$

It has been proved that the RT formula obeys all above properties of the entanglement entropy [8]. However, there is a property possessed by the holographic entanglement entropy (HEE) peculiarly [9-11], that is 


$$
\begin{aligned}
& \text { (5) Monogamy: } S(A)+S(B)+S(C)+S(A B C) \\
& \leq S(A B)+S(A C)+S(B C),
\end{aligned}
$$

which is not obeyed by general quantum states. It gives a constraint on theories that potentially have a holographic duality. Alternatively, properties (1.2)-(1.6) of HEE can be derived by the notion of bit thread [12], an alternative description of the HEE. See further works related to the bit threads in $[13-24]^{1}$.

Moreover, there is a quantity called entanglement of purification (EoP) [25], which is a measure of the classical correlations and quantum entanglement for mixed bipartite states. In [26-28], it has been conjectured that EoP is dual to the area of the minimal cross section on the entanglement wedge [29-31] (and some related works in [32-65]). For two nonoverlapping spatial subregions $A$ and $B$ on the conformal boundary, we have

$$
E_{P}(A: B)=\frac{\operatorname{area}\left(\sigma_{A B}^{\min }\right)}{4 G_{N}},
$$

where $\sigma_{A B}^{\min }$ is the minimal cross section on the entanglement wedge. In $[20,22]$, the bit-thread formulation of the holographic entanglement of purification (HEoP) was given, and many known properties of the HEoP were proved in this formulation. In this paper, however, we would like to go step further and to explore some new properties of HEoP by using bit threads.

According to the statement in [13], we can generalize the flow description of HEE into a meaning of relative homology, while the nesting property of flows goes through as before. Thus, we could carry these flow-based proofs of properties of HEE into homology case, which means we will obtain some corresponding properties in homology case. Remember that the flow description of HEoP in $[20,22]$ is based on the notion of relative homology. We will naturally obtain some corresponding properties for the $\mathrm{HEoP},{ }^{2}$ as

$$
\begin{aligned}
& \text { (1) }\left|E_{P}(A: B C)-E_{P}(B: A C)\right| \leq E_{P}(A B: C), \\
& \text { (2) } E_{P}(A B: C) \leq E_{P}(A: B C)+E_{P}(B: A C), \\
& \text { (3) } E_{P}(B: A C D)+E_{P}(A B C: D) \\
& \quad \leq E_{P}(A B: C D)+E_{P}(B C: A D), \\
& \text { (4) } E_{P}(A: B C D)+E_{P}(C: A B D) \\
& \quad \leq E_{P}(A B: C D)+E_{P}(B C: A D), \\
& \text { (5) } E_{P}(A: B C D)+E_{P}(B: A C D)
\end{aligned}
$$

\footnotetext{
${ }^{1}$ Alternatively, in [66] the authors interpret the RT surface as special Lagrangian cycles calibrated by the real part of the holomorphic oneform of a spacelike hypersurface.

${ }^{2}$ Recently there is a work [40], where new inequalities of HEoP are obtained from HEE, based on the wormhole geometry description of HEoP. While we start from a flow viewpoint, and arrive at a similar conclusion.
}

$$
\begin{aligned}
& +E_{P}(C: A B D)+E_{P}(D: A B C) \\
\leq & E_{P}(A B: C D)+E_{P}(A C: B D) \\
& +E_{P}(B C: A D) .
\end{aligned}
$$

These properties of HEoP obtained from bit threads do not follow from the known properties of EoP. In this paper, we will derive these inequalities by using multiflow description of HEoP defined in relative homology. A geometric analysis is also applied and the validity of these novel inequalities is further confirmed.

This paper is organized as follows: in Sect. 2, we will briefly review the notion of bit threads. Then in Sect. 3, we will have a discussion about generalized HEE defined in relative homology. In Sect. 4.1, we give a multiflow description for HEoP in relative homology. Then in Sect. 4.2, considering tripartite and quadripartite cases, we will derive out some properties of HEoP by using multiflows defined in relative homology, as inequalities (1.8)-(1.12). We notice that each property for HEoP has a corresponding property of HEE in (1.2)-(1.6). A concluding remark is given in the last section.

\section{Review of bit threads}

\subsection{Flow}

The bit threads [12], which are a set of integral curves of a divergenceless norm-bounded vector field $v$ with transverse density equal to $|v|$. The threads of a given vector field are oriented and locally parallel. Consider a manifold $M$ with a conformal boundary $\partial M$, where $A$ is a subregion on $\partial M$ and its complement is $\bar{A}:=\partial M \backslash A$. Define a flow $v_{A \bar{A}}$ with direction from $A$ to $\bar{A}$ on $M$, that is divergenceless and is norm bounded by $1 / 4 G_{N}$ :

$\nabla_{\mu} v_{A \bar{A}}^{\mu}=0, \quad\left|v_{A \bar{A}}\right| \leq \frac{1}{4 G_{N}}$.

As we set the direction for $v_{A \bar{A}}$ flowing from $A$ to $\bar{A}$, it means its flux given by $\int_{A} v_{A \bar{A}}$ is non-negative:

$\int_{A} v_{A \bar{A}}:=\int_{A} \sqrt{h} n_{\mu} v_{A \bar{A}}^{\mu} \geq 0$,

where $h$ is the determinant of the induced metric $h_{i j}$ on $A$ and $n_{\mu}$ is the (inward-pointing) unit normal vector. Then the entanglement entropy between $A$ and $\bar{A}$ is suggested to given by the maximum flux through $A$ among all flows:

$S(A)=\max _{v_{A \bar{A}}} \int_{A} v_{A \bar{A}}$. 


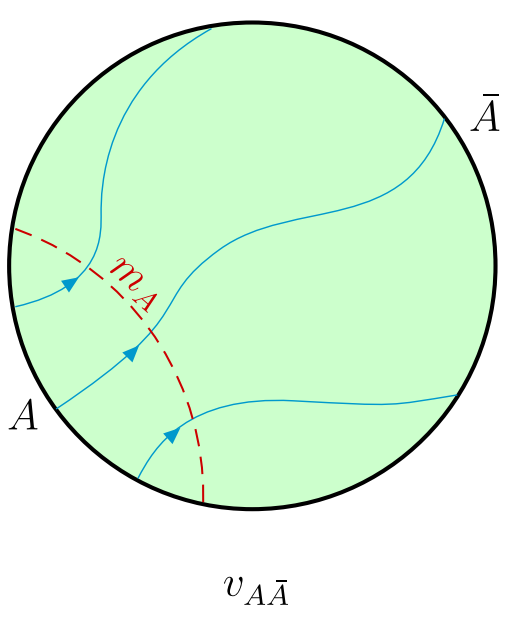

Fig. 1 The vector field $v_{A \bar{A}}$ defined on manifold $M$. The entanglement entropy $S_{A}$ is equal to the maximum flux from $A$ to $\bar{A}$, or equivalently the maximum number of threads connecting $A$ with $\bar{A}$

Equivalence between (2.3) and the RT formula (1.1) is guaranteed by the Riemannian MFMC theorem [12]:

$\max _{v_{A \bar{A}}} \int_{A} v_{A \bar{A}}=\min _{m \sim A} \frac{\operatorname{area}(m)}{4 G_{N}}$.

The left-hand side is a maximum of the flux over all flows $v$, while the right-hand side takes a minimum of the area over all surfaces $m$ homologous to $A$ (written as $m \sim A$ ). The flow interpretation of the holographic entanglement entropy, unlike the minimal surface captured by RT formula jumping under continuous deformations of region $A$ [67-70], varies continuously. And the subadditivity and the strong subadditivity inequalities of HEE can be proved by making use of the properties of flows [12] (Fig. 1).

\subsection{Threads}

In [16], the notion of bit threads was generalized. Instead of being oriented and locally parallel, threads are unoriented and can even intersect with others. The notion of transverse density is replaced by density, defined at a given point on a manifold $M$ as the total length of the threads in a ball of radius $R$ centered on that point divided by the volume of the ball, where $R$ is chosen to be much larger than the Planck scale $G_{N}^{1 /(d-1)}$ and much smaller than the curvature scale of $M$. In the classical or large- $N$ limit $G_{\mathrm{N}} \rightarrow 0$, we can neglect the discretization error between the continuous flow $v$ and the discrete set of threads as the density of threads is large on the scale of $M$.

For region $A$ and its complement $\bar{A}$ on the boundary $\partial M$. Defining a vector field $v_{A \bar{A}}$, we can construct a thread configuration by choosing a set of integral curves with density $\left|v_{A \bar{A}}\right|$. The number of threads $N_{A \bar{A}}$ connecting $A$ to $\bar{A}$ is at least as large as the flux of $v_{A \bar{A}}$ on $A$ :
$N_{A \bar{A}} \geq \int_{A} v_{A \bar{A}}$

Generally, this inequality does not saturate as some of the integral curves may go from $\bar{A}$ to $A$ which have negative contributions to the flux but positive ones to $N_{A \bar{A}}$.

Consider a slab $R$ around $m$, where $R$ is much larger than the Planck length and much smaller than the curvature radius of $M$. The volume of this slab is $R \cdot \operatorname{area}(m)$, the total length of the threads within the slab should be bounded above by $R \cdot \operatorname{area}(m) / 4 G_{N}$. Moreover, any thread connecting $A$ to $\bar{A}$ must have a length within the slab at least $R$. Therefore, we have

$N_{A \bar{A}} \leq \frac{\operatorname{area}(m)}{4 G_{N}}$.

Particularly, for the minimal surface $m_{A}$, we have

$N_{A \bar{A}} \leq \frac{\operatorname{area}\left(m_{A}\right)}{4 G_{N}}=S(A)$.

Combining formulas (2.5) and (2.7), equality holds

$\max N_{A \bar{A}}=\max _{v_{A \bar{A}}} \int_{A} v_{A \bar{A}}=S(A)$.

Thus, $S(A)$ is equal to the maximum number of threads connecting $A$ to $\bar{A}$ over all allowed thread configurations.

\subsection{Multiflow}

The multiflow or multicommodity is the terminology in the network context [71,72]. It is a collection of flows that are compatible with each other, existing on the same geometry simultaneously. It was defined in Riemannian setting to prove the monogamy of mutual information (MMI) in [16]. Consider a Riemannian manifold $M$ with boundary $\partial M$, and let $A_{1}, \ldots, A_{n}$ be non-overlapping regions of $\partial M$, a multiflow is then defined as a set of vector fields $\left\{v_{i j}\right\}$ on $M$ satisfying the following conditions:

$$
\begin{aligned}
v_{i j} & =-v_{j i}, \\
n_{\mu} v_{i j}^{\mu} & =0 \text { on } A_{k}(k \neq i, j), \\
\nabla_{\mu} v_{i j}^{\mu} & =0, \\
\sum_{i<j}^{n}\left|v_{i j}\right| & \leq \frac{1}{4 G_{N}} .
\end{aligned}
$$

There are $n(n-1) / 2$ independent vector fields for the given condition (2.9). Given condition (2.10), $v_{i j}$ is nonvanishing only on $A_{i}$ and $A_{j}$, by (2.11), their flux satisfy

$$
\int_{A_{i}} v_{i j}=-\int_{A_{j}} v_{i j}
$$


Define a vector field

$v_{i \bar{i}}:=\sum_{j \neq i}^{n} v_{i j}$.

The flux of flow $v_{i \bar{i}}$ should be bounded above by the entropy of $A_{i}$ :

$\int_{A_{i}} v_{i \bar{i}} \leq S\left(A_{i}\right)$.

The inequality will saturate for a max flow. Given $v_{i j}(i<j)$, we can choose a set of threads with density $\left|v_{i j}\right|$. From (2.5), the number of threads connect $A_{i}$ to $A_{j}$ is at least the flux of $v_{i j}$ :

$N_{A_{i} A_{j}} \geq \int_{A_{i}} v_{i j}$.

Summing (2.16) over $j \neq i$ for fixed $i$, we have

$\sum_{j \neq i}^{n} N_{A_{i} A_{j}}=N_{A_{i} \bar{A}_{i}} \geq \int_{A_{i}} v_{i \bar{i}}$.

On the other hand, (2.7) implies that the total number of threads emerging out of $A_{i}$ is bounded above by $S\left(A_{i}\right)$ :

$\sum_{j \neq i}^{n} N_{A_{i} A_{j}}=N_{A_{i} \bar{A}_{i}} \leq S\left(A_{i}\right)$.

Therefore, both inequalities (2.17) and (2.19) saturate for a max flow with fixed $i$ :

$\sum_{j \neq i}^{n} N_{A_{i} A_{j}}=N_{A_{i} \bar{A}_{i}}=\int_{A_{i}} v_{i \bar{i}}=S\left(A_{i}\right)$.

Furthermore, the inequality (2.16) must be individually saturated:

$N_{A_{i} A_{j}}=\int_{A_{i}} v_{i j}$.

The above discussion focuses only on the case for a fixed $i$. Remarkably, it has been proved in [16] that there exists a socalled max multiflow $\left\{v_{i j}\right\}$ saturating all $n$ bounds in (2.15) simultaneously. Or equivalently, there exists a so-called $\max$ thread configuration in the language of threads, as the formula (2.19) holding for all $i$.

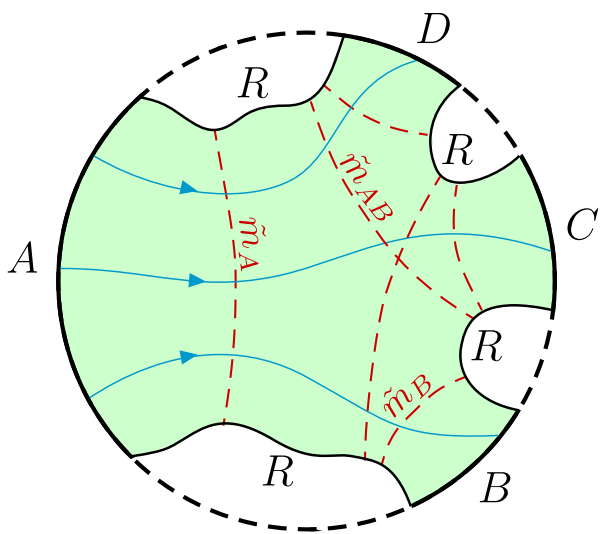

v

Fig. 2 The vector field $v$ is defined on $M^{\prime}$, the region surrounded by $A \cup B \cup C \cup D \cup R$, as we have imposed a no-flux condition on surface $R$. And $\tilde{m}_{A}, \tilde{m}_{B}$ and $\tilde{m}_{A B}$ are the minimal surfaces homologous to regions $A, B$ and $A B$ relative to $R$, respectively

\section{Relative homology and generalized HEE}

The notion of relative homology was introduced to generalize the MFMC theorem in [13]. To get an intuition for the generalized MFMC (gMFMC) theorem, we consider a manifold $M$ with a conformal boundary $\partial M$ and $A$ is a subregion on the boundary. Specifically, let $R$ be a bulk surface attached to the boundary, for instance as Fig. 2. For region $A$, we can define the surface $\tilde{m}$ homologous to $A$ relative to $R$ (written as $\tilde{m} \sim A \operatorname{rel} R$ ), where surface $\tilde{m}$ is allowed to begin and end on $R .{ }^{3}$ On flow side, this corresponds to imposing a Neumann condition (no-flux condition) on $R$, thereby

$\nabla_{\mu} v^{\mu}=0,|v| \leq \frac{1}{4 G_{N}}, n_{\mu} v^{\mu}=0$ on $R$

This means no flux through $R$. Therefore, the flow is restricted within region $M^{\prime}$ with boundary $\partial M^{\prime}=A \cup B \cup$ $C \cup D \cup R$. Finally, we can arrive at the gMFMC theorem

$\max _{\substack{\nu: \\\left.n_{\mu} v^{\mu}\right|_{R}=0}} \int_{A} v=\min _{\substack{\tilde{\tilde{m}} \sim A \\ r e l} \text { on } M} \frac{\operatorname{area}(\tilde{m})}{4 G_{N}}=\frac{\operatorname{area}\left(\tilde{m}_{A}\right)}{4 G_{N}}$,

where $\tilde{m}_{A}$ is the minimal surface homologous to $A$ relative to $R$ on region $M^{\prime}$. The flow description of HEE in [12] is specifically based on the homology relative to $R=\partial A$. As proposed in [13], we will have a generalized HEE

$\tilde{S}(A):=\max _{\substack{v: \\\left.n_{\mu} \nu^{\mu}\right|_{R}=0}} \int_{A} v$.

\footnotetext{
${ }^{3}$ Here we use the notion $\tilde{m}$ defined on relative homology region $M^{\prime}$, to distinguish from the surface $m$ defined on original manifold $M$.
} 
This notion originates from HEE, but defined in a meaning of general relative homology. We stress that, differing from $\mathrm{HEE}$, this quantity has no clear quantum information meaning generally in holography. However, there is another quantity with the form (3.3) arousing our interests in holography, i.e. HEoP. Similar to HEE, the HEoP can also be regarded as a special case in (3.3) with the homology relative to $R=m_{A B}$ $[20,22]$.

The formula (3.2) establishes the equivalence between the flow objects and geometric objects in holography. We have learned that we could derive the properties of holographic objects from the properties of flows, such as nesting of flows. Remember the flow-based proofs of the Araki-Lieb (AL) inequality, the subadditivity (SA) and the strong subadditivity (SSA) for the HEE [12], also the multiflow-based proof of the monogamy of the mutual information (MMI) [16, 17]. Note that when the min cut is defined in homology relative to a bulk boundary $R$, the dual flow (or multiflow) is subjected to a no-flux boundary condition on $R$. The nesting property of flows in relative homology goes through as before [13]. Thus these flow-based proofs for properties of HEE could be carried into the relative homology cases in similar manner, meanwhile what we need to do on geometric side is just replacing with the cuts defined in relative homology. Namely, based on the flow viewpoints, we argue that the properties of HEE will hold for quantity $\tilde{S}$ generally (including HEoP case), as

$$
\begin{aligned}
& |\tilde{S}(A)-\tilde{S}(B)| \leq \tilde{S}(A B) \leq \tilde{S}(A)+\tilde{S}(B), \\
& \tilde{S}(B)+\tilde{S}(A B C) \leq \tilde{S}(A B)+\tilde{S}(B C) \\
& \tilde{S}(A)+\tilde{S}(C) \leq \tilde{S}(A B)+\tilde{S}(B C) \\
& \tilde{S}(A)+\tilde{S}(B)+\tilde{S}(C)+\tilde{S}(A B C) \\
& \quad \leq \tilde{S}(A B)+\tilde{S}(A C)+\tilde{S}(B C)
\end{aligned}
$$

corresponding to the inequalities (1.2)-(1.6) of HEE. ${ }^{4}$ It seems that these inequalities could be intuitively obtained geometrically by making use of the minimality of relative homology surface. For example, as $A B \sim \tilde{m}_{A} \cup \tilde{m}_{B} \sim$ $\tilde{m}_{A B}$ rel $R$, due to the minimality of $\tilde{m}_{A B}$ among all surfaces homologous to $A B$ relative to $R$, we naturally obtain $\left|\tilde{m}_{A B}\right| \leq\left|\tilde{m}_{A}\right|+\left|\tilde{m}_{B}\right|$. As to $A \sim \tilde{m}_{A} \sim \tilde{m}_{A B} \cup \tilde{m}_{B}$ rel $R$, due to the minimality of $\tilde{m}_{A}$ among all surfaces homologous to $A$ relative to $R$, we have $\left|\tilde{m}_{A}\right| \leq\left|\tilde{m}_{A B}\right|+\left|\tilde{m}_{B}\right|$. In the following, we will focus on the multiflow-based proofs of these inequalities for HEoP.

\footnotetext{
${ }^{4}$ We emphasize that we only lists the inequalities of $\tilde{S}$ corresponding to some basic inequalities of HEE here. While our argument based on flow viewpoints allows us to reap more once we take other inequalities of HEE into consideration.
}

\section{Properties of HEoP derived from bit threads}

\subsection{Multiflow description of HEoP as relative homology}

The bit thread formulation of the HEoP has been given in $[20,22]$, which is based on the gMFMC theorem. In this section, instead, we would like to apply the notion of multiflow defined in relative homology, to give a multiflow description for HEoP. Taking a manifold $M$ with non-overlapping subregions $A_{1}, \ldots, A_{n}$ on boundary $\partial M$. Consider the entanglement wedge $r_{A_{1} A_{2} \ldots A_{n}}$ with boundary $\partial r_{A_{1} A_{2} \ldots A_{n}}=\mathcal{A} \cup$ $m_{A_{1} A_{2} \ldots A_{n}}$, where $\mathcal{A}:=A_{1} \cup A_{2} \cup \cdots \cup A_{n}$. We could define a multiflow $\left\{v_{i j}\right\}$ on the entanglement wedge, subject to a Neumann boundary condition on $m_{A_{1} A_{2} \ldots A_{n}}$. Namely,

$$
\begin{aligned}
v_{i j} & =-v_{j i}, \\
n_{\mu} v_{i j}^{\mu} & =0 \text { on } m_{A_{1} A_{2} \ldots A_{n},} \\
n_{\mu} v_{i j}^{\mu} & =0 \text { on } A_{k}(k \neq i, j), \\
\nabla_{\mu} v_{i j}^{\mu} & =0 \\
\sum_{i<j}^{n}\left|v_{i j}\right| & \leq \frac{1}{4 G_{N}} .
\end{aligned}
$$

This means no flux through $m_{A_{1} A_{2} \ldots A_{n}}$ or no threads connecting to $m_{A_{1} A_{2} \ldots A_{n}}$. In this way, we restrict the multiflow inside geometry $r_{A_{1} A_{2} \ldots A_{n}}$. All following discussions will be based on such a multiflow configuration.

We set the direction of $v_{i j}$ as a flow from $A_{i}$ to $A_{j}$, which means the flux $\int_{A_{i}} v_{i j}$ out of $A_{i}$ (inward-pointing on $A_{i}$ ) is non-negative:

$\int_{A_{i}} v_{i j}:=\int_{A_{i}} \sqrt{h} n_{\mu} v_{i j}^{\mu} \geq 0$,

where $h$ is the determinant of the induced metric on $A$ and $n$ is chosen to be a (inward-pointing) unit normal vector. Given condition (4.2) and (4.3) the fact that $v_{i j}$ is non-vanishing only on $A_{i}$ and $A_{j}$, combining (4.1) and (4.4), we get

$\int_{A_{i}} v_{i j}=-\int_{A_{i}} v_{j i}=\int_{A_{j}} v_{j i} \geq 0$.

Similarly as before, we can define a minimal cut $\tilde{m}_{A_{i}}$ on $r_{A_{1} A_{2} \ldots A_{n}}$ homologous to region $A_{i}$ relative to $m_{A_{1} A_{2} \ldots A_{n}}$, which is exactly the minimal cross section $\sigma_{A_{i}}^{\text {min } \bar{A}_{i}}$ where $\bar{A}_{i}:=$ $\mathcal{A} \backslash A_{i}$. Here, the quantity $\tilde{S}$ is just $E_{P}$ as proposed in [20,22]. The dual flow is defined as

$v_{i \bar{i}}:=\sum_{j \neq i}^{n} v_{i j}$

The flux of flow $v_{i \bar{i}}$ should be bounded above by the area of minimal cross section $\sigma_{A_{i} \bar{A}_{i}}^{\min }$. Combining with the conjecture 
of $E_{P}=E_{W}$, we have

$$
\int_{A_{i}} v_{i \bar{i}} \leq \min _{\substack{\sigma_{A_{i} \bar{A}_{i}} \sim A_{i} \\ r e l m_{A_{1} A_{2} \ldots A_{n}}}} \frac{\operatorname{area}\left(\sigma_{A_{i} \bar{A}_{i}}\right)}{4 G_{N}}=E_{P}\left(A_{i}: \bar{A}_{i}\right) .
$$

In addition, the number of threads connect $A_{i}$ to $A_{j}$ is at least the flux of $v_{i j}$ :

$N_{A_{i} A_{j}} \geq \int_{A_{i}} v_{i j}$

Let us consider a max multiflow (or equivalently max thread configuration), as introduced in Sect. 2.3, the inequality in (4.9) saturates simultaneously for all $i$, thus

$\sum_{j \neq i}^{n} N_{A_{i} A_{j}}=N_{A_{i} \bar{A}_{i}}=\int_{A_{i}} v_{i \bar{i}}=E_{P}\left(A_{i}: \bar{A}_{i}\right)$,

Furthermore, all inequalities (4.10) individually saturated:

$N_{A_{i} A_{j}}=\int_{A_{i}} v_{i j}$

The bipartite case has been shown in [20,22]. In what follows, we will study such a max multiflow configuration (or equivalently max thread configuration) for tripartite and quadripartite cases, to derive out some new inequalities of the HEoP. As we will show that for each property (considering only AL, SA, SSA, MMI) of HEE that can be derived from bit threads, there is a corresponding property for HEoP.

\subsection{Properties of HEoP derived from bit threads}

\subsubsection{Tripartite case}

Consider a max multifow (or max thread configuration) for tripartite case with completely connected phase ${ }^{5}$, as shown in Fig. 3. By (4.11) and (4.12), we have

\footnotetext{
5 Note that there are two other disconnected phases of region $r_{A B C}$. Subject to the no-flux condition on $m_{A B C}$, the disconnected phase means no-flow (or no-thread) passing between disconnected regions. Here, we just focus on completely connected case. Similar consideration is adopted for quadripartite case.
}

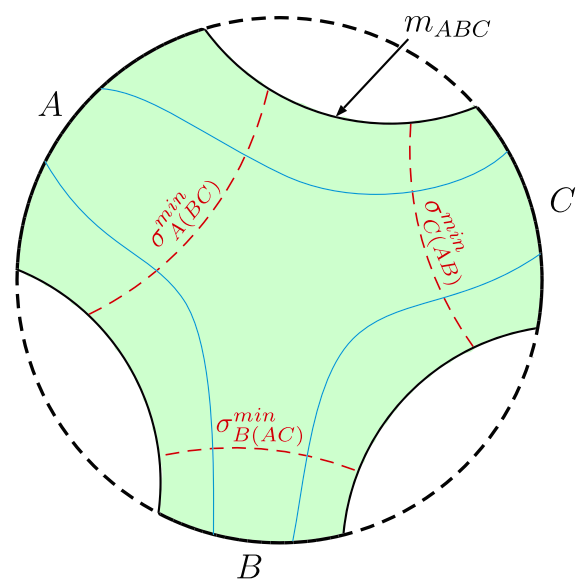

max thread configuration on $r_{A B C}$

Fig. 3 The max thread configuration for tripartite case. Threads are unoriented and can intersect with others. The number of threads connecting to each single region reaches its maximum value

$$
\begin{aligned}
E_{P}(A: B C)= & \int_{A} v_{A(B C)}=\int_{A} v_{A B} \\
& +\int_{A} v_{A C}=N_{A B}+N_{A C}, \\
E_{P}(B: A C)= & \int_{B} v_{B(A C)}=\int_{B} v_{B A} \\
& +\int_{B} v_{B C}=N_{B A}+N_{B C}, \\
E_{P}(C: A B)= & \int_{C} v_{C(A B)}=\int_{C} v_{C A} \\
& +\int_{C} v_{C B}=N_{C A}+N_{C B} .
\end{aligned}
$$

Then from (4.13) we obtain

$$
\begin{aligned}
& E_{P}(A: B C)+E_{P}(B: A C)-E_{P}(A B: C) \\
& \quad=2 \int_{A} v_{A B}=2 N_{A B}, \\
& E_{P}(A B: C)-\left(E_{P}(A: B C)-E_{P}(B: A C)\right) \\
& \quad=2 \int_{B} v_{B C}=2 N_{B C}, \\
& E_{P}(A B: C)-\left(E_{P}(B: A C)-E_{P}(A: B C)\right) \\
& \quad=2 \int_{A} v_{A C}=2 N_{A C},
\end{aligned}
$$

where the relations (4.7) are used. Due to the non-negativity of the number of threads, we immediately get the inequalities

$$
\begin{aligned}
& \left|E_{P}(A: B C)-E_{P}(B: A C)\right| \leq E_{P}(A B: C) \\
& \quad \leq E_{P}(A: B C)+E_{P}(B: A C) .
\end{aligned}
$$


The first inequality can also be obtained from the second inequality by alternating labels $\mathrm{A}, \mathrm{B}$ and $\mathrm{C}$. The inequalities (4.15) exactly correspond to the inequalities (3.4) for HEoP case, i.e. the AL inequality and the SA. This property of HEoP has already been derived in $[20,40]$. It does not follow by the known properties of EoP.

Moreover, this can also be intuitively obtained from geometric side by comparing with the cuts defined in relative homology, as shown in Fig. 3. As $A B \sim \sigma_{A(B C)}^{\min } \cup \sigma_{B(A C)}^{\min } \sim$ $\sigma_{(A B) C}^{\min } \sim C$ rel $m_{A B C}$, due to the minimality of $\sigma_{(A B) C}^{\min }$ among all surfaces homologous to $A B$ relative to $m_{A B C}$, we naturally obtain the area relation $\left|\sigma_{(A B) C}^{\min }\right| \leq\left|\sigma_{A(B C)}^{\min }\right|+$ $\left|\sigma_{B(A C)}^{\min }\right|$, that is, $E_{P}(A B: C) \leq E_{P}(A: B C)+E_{P}(B:$ $A C)$ by HEoP conjecture. Similarly, as $A \sim \sigma_{A(B C)}^{\min } \sim$ $\sigma_{B(A C)}^{\min } \cup \sigma_{(A B) C}^{\min } \sim B C$ rel $m_{A B C}$, due to the minimality of $\sigma_{A(B C)}^{\min }$ among all surfaces homologous to $A$ relative to $m_{A B C}$, we get the inequality $\left|\sigma_{A(B C)}^{\min }\right| \leq\left|\sigma_{B(A C)}^{\min }\right|+$ $\left|\sigma_{(A B) C}^{\min }\right|$, that is, $E_{P}(A: B C) \leq E_{P}(B: A C)+E_{P}$ $(A B: C)$.

\subsubsection{Quadripartite case}

Consider a max multifow (or max thread configuration) for quadripartite case as sketched in Fig. 4. From (4.11) and (4.12), we have

$$
\begin{aligned}
E_{P}(A: B C D)= & \int_{A} v_{A(B C D)}=\int_{A} v_{A B}+\int_{A} v_{A C} \\
& +\int_{A} v_{A D}=N_{A B}+N_{A C}+N_{A D}, \\
E_{P}(B: A C D)= & \int_{B} v_{B(A C D)}=\int_{B} v_{B A}+\int_{B} v_{B C} \\
& +\int_{B} v_{B D}=N_{B A}+N_{B C}+N_{B D}, \\
E_{P}(C: A B D)= & \int_{C} v_{C(A B D)}=\int_{C} v_{C A}+\int_{C} v_{C B} \\
& +\int_{C} v_{C D}=N_{C A}+N_{C B}+N_{C D}, \\
E_{P}(D: A B C)= & \int_{D} v_{D(A B C)}=\int_{D} v_{D A}+\int_{D} v_{D B} \\
& +\int_{D} v_{D C}=N_{D A}+N_{D B}+N_{D C} .
\end{aligned}
$$

Noting that for a max multifow configuration, the flux through the union regions $A B, A C$ and $B C$ (or equivalently the number of threads emerging from these union regions) cannot reach its maximum value in general. Thus

$$
\begin{aligned}
E_{P}(A B: C D) & \geq \int_{A B} v_{(A B)(C D)} \\
& =\int_{A} v_{A C}+\int_{A} v_{A D}+\int_{B} v_{B C}+\int_{B} v_{B D} \\
& =N_{A C}+N_{A D}+N_{B C}+N_{B D}, \\
E_{P}(A C: B D) & \geq \int_{A C} v_{(A C)(B D)} \\
& =\int_{A} v_{A B}+\int_{A} v_{A D}+\int_{C} v_{C B}+\int_{C} v_{C D} \\
& =N_{A B}+N_{A D}+N_{C B}+N_{C D}, \\
E_{P}(B C: A D) & \geq \int_{B C} v_{(B C)(A D)} \\
& =\int_{B} v_{B A}+\int_{B} v_{B D}+\int_{C} v_{C A}+\int_{C} v_{C D} \\
& =N_{B A}+N_{B D}+N_{C A}+N_{C D} .
\end{aligned}
$$

Now from (4.16) and (4.17), and combining with relations (4.7), we obtain the inequality

$$
\begin{aligned}
E_{P} & (A B: C D)+E_{P}(B C: A D) \\
& -E_{P}(B: A C D)-E_{P}(A B C: D) \\
\geq & \int_{A B} v_{(A B)(C D)}+\int_{B C} v_{(B C)(A D)} \\
& -\int_{B} v_{B(A C D)}-\int_{A B C} v_{(A B C) D} \\
= & 2 \int_{A} v_{A C}=2 N_{A C} \geq 0,
\end{aligned}
$$

and the inequality

$$
\begin{aligned}
E_{P} & (A B: C D)+E_{P}(B C: A D) \\
& -E_{P}(A: B C D)-E_{P}(C: A B D) \\
\geq & \int_{A B} v_{(A B)(C D)}+\int_{B C} v_{(B C)(A D)} \\
& -\int_{A} v_{A(B C D)}-\int_{C} v_{C(A B D)} \\
= & 2 \int_{B} v_{B D}=2 N_{B D} \geq 0 .
\end{aligned}
$$

Furthermore, we obtain that

$$
\begin{aligned}
& E_{P}(A B: C D)+E_{P}(A C: B D)+E_{P}(B C: A D) \\
& \quad-E_{P}(A: B C D)-E_{P}(B: A C D) \\
& \quad-E_{P}(C: A B D)-E_{P}(D: A B C) \\
& \geq \int_{A B} v_{(A B)(C D)}+\int_{A C} v_{(A C)(B D)}+\int_{B C} v_{(B C)(A D)} \\
& \quad-\int_{A} v_{A(B C D)}-\int_{B} v_{B(A C D)}-\int_{C} v_{C(A B D)} \\
& \quad-\int_{D} v_{D(A B C)}=0 .
\end{aligned}
$$




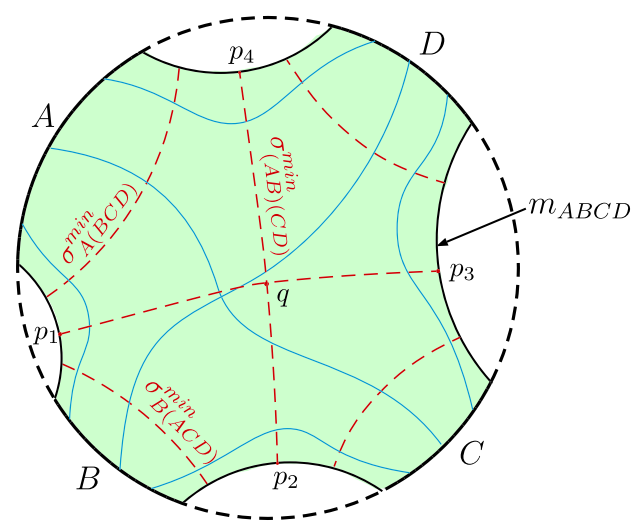

max thread configuration on $r_{A B C D}$

Fig. 4 The max thread configuration for quadripartite case. Threads are unoriented and can intersect with others. The number of threads connecting to each single region reaches its maximum value

Here we obtain the inequalities (4.18), (4.19) and (4.20) by multiflows, which respectively corresponds to (3.5), (3.6) and (3.7) for HEoP case. As far as we know, these properties of the HEoP are also new, and are out of the known properties of the EoP [25]. It is worth to explore whether these properties are held by EoP for generic quantum states, or only valid for the holographic quantum states that have classical gravity duality.

Again, we can verify these properties from the geometric side. To do this, let us compare with these cuts defined in relative homology. First, we divide the union surface $\sigma_{(A B)(C D)}^{\min } \cup \sigma_{(B C)(A D)}^{\min }$ into four parts $\left(p_{1} q\right),\left(p_{2} q\right),\left(p_{3} q\right)$ and $\left(p_{4} q\right)$, as shown in Fig. 4. Since $B \sim \sigma_{B(A C D)}^{\min } \sim$ $\left(p_{1} q\right) \cup\left(p_{2} q\right)$ and $D \sim \sigma_{(A B C) D}^{\min } \sim\left(p_{3} q\right) \cup\left(p_{4} q\right)$ relative to $m_{A B C D}$, and considering the minimality of $\sigma_{B(A C D)}^{\min }$ and $\sigma_{(A B C) D}^{\min }$, we have

$$
\begin{aligned}
& \left|\sigma_{B(A C D)}^{\min }\right|+\left|\sigma_{(A B C) D}^{\min }\right| \leq\left|\left(p_{1} q\right)\right|+\left|\left(p_{2} q\right)\right| \\
& \quad+\left|\left(p_{3} q\right)\right|+\left|\left(p_{4} q\right)\right|=\left|\sigma_{(A B)(C D)}^{\min }\right|+\left|\sigma_{(B C)(A D)}^{\min }\right|,
\end{aligned}
$$

which is corresponding to the inequality (4.18) by HEoP conjecture. Similar analysis leads to inequality (4.19).

As to inequality (4.20), we notice that $\sigma_{(A C)(B D)}^{\min }=$ $\min \left\{\sigma_{A(B C D)}^{\min } \cup \sigma_{C(A B D)}^{\min }, \sigma_{B(A C D)}^{\min } \cup \sigma_{(A B C) D}^{\min }\right\}$ for oppositeposition regions. We then can choose that $\left|\sigma_{A(B C D)}^{\min }\right|+$ $\left|\sigma_{C(A B D)}^{\min }\right| \leq\left|\sigma_{B(A C D)}^{\min }\right|+\left|\sigma_{(A B C) D}^{\min }\right|$. In the end we obtain

$$
\begin{aligned}
& \left|\sigma_{(A B)(C D)}^{\min }\right|+\left|\sigma_{(A C)(B D)}^{\min }\right|+\left|\sigma_{(B C)(A D)}^{\min }\right|-\left|\sigma_{A(B C D)}^{\min }\right| \\
& \quad-\left|\sigma_{B(A C D)}^{\min }\right|-\left|\sigma_{C(A B D)}^{\min }\right|-\left|\sigma_{(A B C) D}^{\min }\right| \\
& =\left|\sigma_{(A B)(C D)}^{\min }\right|+\left|\sigma_{(B C)(A D)}^{\min }\right|
\end{aligned}
$$

$$
-\left|\sigma_{B(A C D)}^{\min }\right|-\left|\sigma_{(A B C) D}^{\min }\right| .
$$

Thus the area relation will reduce to the case for inequality (4.18) or (4.19), which we have proved in the previous paragraph. Finally, we finish the proofs from geometric side.

\section{Entropy cone for HEoP}

Section 3 suggests a correspondence between the properties of the HEE and the ones of the HEoP. On the other hand, the work in [73], which defines the holographic entropy cone of all entanglement entropy vectors for the holographic quantum states (see [74-79] for more recent progress), reminds us to discuss the possibility to define an entropy cone for the holographic entanglement of purification. Particularly, we can likewise define an entropy cone for HEoP in terms of these inequalities (1.8)-(1.12) derived in this paper. We notice that each inequality obtained for HEoP has the same form as the corresponding inequality of HEE. Thus the entropy cone of HEoP defined here will have the same structure as the one of HEE, except that it is defined in the entropy space of entanglement of purification.

For a tripartite state $\rho_{A B C}$, we choose $\left\{E_{P}(A: B C), E_{P}(B\right.$ : $\left.A C), E_{P}(A B: C)\right\}$ as the basis of the entropy space. And the extreme ray of the HEoP cone is

$\left(E_{P}(A: B C), E_{P}(B: A C), E_{P}(A B: C)\right)=(1,1,0)$

up to the permutation symmetry. It reduces to the case of $\mathcal{C}_{2}$ of HEE when $\rho_{A B C}$ is pure, as $E_{P}(A: B C)=S(A), E_{P}(B$ : $A C)=S(B), E_{P}(A B: C)=S(A B)$.

For a quadripartite state $\rho_{A B C D}$, after choosing the following basis, the extreme ray (up to the permutation symmetry) then is given by

$$
\begin{aligned}
& \left(E_{P}(A: B C D), E_{P}(B: A C D), E_{P}(C: A B D),\right. \\
& \quad E_{P}(D: A B C) ; E_{P}(A B: C D), \\
& \left.E_{P}(A C: B D), E_{P}(B C: A D)\right)=(1,1,1,1 ; 2,2,2) .
\end{aligned}
$$

It reduces to the case of $\mathcal{C}_{3}$ of $\mathrm{HEE}$ when $\rho_{A B C D}$ is pure, as these HEoP sets are equal to HEE sets.

Furthermore, as stating in Sect. 3, we can further argue that, for each inequality of HEE there always exists a corresponding inequality for HEoP, as it can be likewise proved by bit threads in the relative homology case. If this is the case, it will help us define the entropy cone of HEoP for more partite cases by considering other inequalities of HEE. Moreover, besides these inequalities expected from HEE according to our argument, there are also other known inequalities 
for HEoP [26-28]. Further study is needed to show whether these inequalities could together define a tighter entropy cone for HEoP to make constraints on the allowable holographic quantum states. We leave this discussion for the future work.

\section{Conclusion}

In this paper, we obtain some new properties (1.8)-(1.12) of the HEoP that corresponds, respectively, to AL, SA, SSA and MMI by utilizing the multiflow description of the HEoP. Thus, starting from flow viewpoints, we arrive at a similar conclusion as in [40], and we give the multiflow-based derivations of these new inequalities. Note that there are several other quantum counterparts relating to the $E_{W}$, such as logarithmic negativity [37,54], odd entropy [38], reflected entropy [51] and $R$-correlation [55,58]. These new inequalities of HEoP should be also valid for other feasible counterparts, and make some constraints on these feasible candidates of $E_{W}$. Intuitively, these inequalities can also be obtained from geometric side by comparing with the cuts defined in relative homology for tripartite and quadripartite cases. But usually it could be more complicated and less obvious to find the inequalities of HEoP hiding in more partite cases from geometric side.

We can even go further, and conjecture that: For each property of HEE that can be derived from bit threads, there always exists a corresponding property for HEoP that can be obtained from bit threads defined in relative homology. The opposite is not true. We explain that all the flow-based proofs of the properties of HEE, can be carried into the relative homology cases in a similar manner, while on geometric side we just need to replace with the cuts defined in relative homology. Thus we could finally obtain some corresponding properties for HEoP. This is remarkable if it is true. As it allows one to explore much more constraints of $E_{W}$ by associating with the contents of holographic entropy cone. We leave the proof of this conjecture as a challenge for the future.

Acknowledgements We would like to thank Matt Headrick for useful discussion. This work was supported in part by the National Natural Science Foundation of China under Grant numbers 11975116, 11665016 , 11565019 and 11563006, and Jiangxi Science Foundation for Distinguished Young Scientists under Grant number 20192BCB23007.

Data Availability Statement This manuscript has no associated data or the data will not be deposited. [Authors' comment: The paper does not use any data].

Open Access This article is licensed under a Creative Commons Attribution 4.0 International License, which permits use, sharing, adaptation, distribution and reproduction in any medium or format, as long as you give appropriate credit to the original author(s) and the source, provide a link to the Creative Commons licence, and indicate if changes were made. The images or other third party material in this article are included in the article's Creative Commons licence, unless indicated otherwise in a credit line to the material. If material is not included in the article's Creative Commons licence and your intended use is not permitted by statutory regulation or exceeds the permitted use, you will need to obtain permission directly from the copyright holder. To view a copy of this licence, visit http://creativecomm ons.org/licenses/by/4.0/.

Funded by SCOAP $^{3}$.

\section{References}

1. G't Hooft, Dimensional reduction in quantum gravity. Conf. Proc. C 930308, 284 (1993). arXiv:gr-qc/9310026

2. L. Susskind, The World as a hologram. J. Math. Phys. 36, 6377 (1995). arXiv:hep-th/9409089

3. J.M. Maldacena, The Large $\mathrm{N}$ limit of superconformal field theories and supergravity. Adv. Theor. Math. Phys. 2, 231 (1998). arXiv:hep-th/9711200

4. E. Witten, Anti-de Sitter space and holography. Adv. Theor. Math. Phys. 2, 253 (1998). arXiv:hep-th/9802150

5. S.S. Gubser, I.R. Klebanov, A.M. Polyakov, Gauge theory correlators from noncritical string theory. Phys. Lett. B 428, 105 (1998). arXiv:hep-th/9802109

6. S. Ryu, T. Takayanagi, Holographic derivation of entanglement entropy from AdS/CFT. Phys. Rev. Lett. 96, 181602 (2006). arXiv:hep-th/0603001

7. V.E. Hubeny, M. Rangamani, T. Takayanagi, A Covariant holographic entanglement entropy proposal. JHEP 0707, 062 (2007). arXiv:0705.0016 [hep-th]

8. M. Headrick, T. Takayanagi, A Holographic proof of the strong subadditivity of entanglement entropy. Phys. Rev. D 76, 106013 (2007). arXiv:0704.3719 [hep-th]

9. P. Hayden, M. Headrick, A. Maloney, Holographic mutual information is monogamous. Phys. Rev. D 87(4), 046003 (2013). arXiv: 1107.2940 [hep-th]

10. V. Balasubramanian, A. Bernamonti, N. Copland, B. Craps, F. Galli, Thermalization of mutual and tripartite information in strongly coupled two dimensional conformal field theories. Phys. Rev. D 84, 105017 (2011). arXiv: 1110.0488 [hep-th]

11. A. Allais, E. Tonni, Holographic evolution of the mutual information. JHEP 1201, 102 (2012). arXiv: 1110.1607 [hep-th]

12. M. Freedman, M. Headrick, Bit threads and holographic entanglement. Commun. Math. Phys. 352(1), 407 (2017). arXiv:1604.00354 [hep-th]

13. M. Headrick, V.E. Hubeny, Riemannian and Lorentzian flow-cut theorems. Class. Quant. Grav. 35(10), 10 (2018). arXiv:1710.09516 [hep-th]

14. C.B. Chen, F.W. Shu, M.H. Wu, Quantum bit threads of MERA tensor network in large $c$ limit. arXiv:1804.00441 [hep-th]

15. J. Harper, M. Headrick, A. Rolph, Bit Threads in Higher Curvature Gravity. JHEP 1811, 168 (2018). arXiv:1807.04294 [hep-th]

16. S.X. Cui, P. Hayden, T. He, M. Headrick, B. Stoica, M. Walter, Bit Threads and Holographic Monogamy Commun. Math. Phys. (2019). arXiv:1808.05234 [hep-th]

17. V.E. Hubeny, Bulk locality and cooperative flows. JHEP 1812, 068 (2018). arXiv:1808.05313 [hep-th]

18. C.A. Agón, J. De Boer, J.F. Pedraza, Geometric aspects of holographic bit threads. JHEP 1905, 075 (2019). arXiv:1811.08879 [hep-th]

19. J. Kudler-Flam, I. MacCormack, S. Ryu, Holographic entanglement contour, bit threads, and the entanglement tsunami. J. Phys. A 52(32), 325401 (2019). arXiv:1902.04654 [hep-th] 
20. D.H. Du, C.B. Chen, F.W. Shu, Bit threads and holographic entanglement of purification. JHEP 1908, 140 (2019). arXiv:1904.06871 [hep-th]

21. N. Bao, A. Chatwin-Davies, J. Pollack, G.N. Remmen, Towards a bit threads derivation of holographic entanglement of purification. JHEP 1907, 152 (2019). arXiv:1905.04317 [hep-th]

22. J. Harper, M. Headrick, Bit threads and holographic entanglement of purification. JHEP 1908, 101 (2019). arXiv: 1906.05970 [hep-th]

23. C.A. Agón, M. Mezei, Bit threads and the membrane theory of entanglement dynamics. arXiv:1910.12909 [hep-th]

24. J. Harper, Multipartite entanglement and topology in holography. arXiv:2006.02899 [hep-th]

25. B.M. Terhal, M. Horodecki, D.W. Leung, D.P. DiVincenzo, The entanglement of purification. J. Math. Phys. 43, 4286 (2002). arXiv:quant-ph/0202044

26. K. Umemoto, T. Takayanagi, Entanglement of purification through holographic duality. Nat. Phys. 14(6), 573 (2018). arXiv:1708.09393 [hep-th]

27. P. Nguyen, T. Devakul, M.G. Halbasch, M.P. Zaletel, B. Swingle, Entanglement of purification: from spin chains to holography. JHEP 1801, 098 (2018). arXiv:1709.07424 [hep-th]

28. N. Bao, I.F. Halpern, Holographic Inequalities and Entanglement of Purification. JHEP 1803, 006 (2018). arXiv: 1710.07643 [hep-th]

29. B. Czech, J.L. Karczmarek, F. Nogueira, M. Van Raamsdonk, The Gravity Dual of a Density Matrix. Class. Quant. Grav. 29, 155009 (2012). arXiv:1204.1330 [hep-th]

30. A.C. Wall, Maximin Surfaces, and the Strong Subadditivity of the Covariant Holographic Entanglement Entropy. Class. Quant. Grav. 31(22), 225007 (2014). arXiv:1211.3494 [hep-th]

31. M. Headrick, V.E. Hubeny, A. Lawrence, M. Rangamani, Causality and holographic entanglement entropy. JHEP 1412, 162 (2014). arXiv:1408.6300 [hep-th]

32. A. Bhattacharyya, T. Takayanagi, K. Umemoto, Entanglement of purification in free scalar field theories. JHEP 1804, 132 (2018). arXiv:1802.09545 [hep-th]

33. H. Hirai, K. Tamaoka, T. Yokoya, Towards entanglement of purification for conformal field theories. PTEP 2018(6), 063B03 (2018). arXiv:1803.10539 [hep-th]

34. R. Espíndola, A. Guijosa, J.F. Pedraza, Entanglement wedge reconstruction and entanglement of purification. Eur. Phys. J. C 78(8), 646 (2018). arXiv:1804.05855 [hep-th]

35. N. Bao, I.F. Halpern, Conditional and multipartite entanglements of purification and holography. Phys. Rev. D 99(4), 046010 (2019). arXiv: 1805.00476 [hep-th]

36. K. Umemoto, Y. Zhou, Entanglement of purification for multipartite states and its holographic dual. JHEP 1810, 152 (2018). arXiv: 1805.02625 [hep-th]

37. J. Kudler-Flam, S. Ryu, Entanglement negativity and minimal entanglement wedge cross sections in holographic theories. Phys. Rev. D 99(10), 106014 (2019). arXiv:1808.00446 [hep-th]

38. K. Tamaoka, Entanglement wedge cross section from the dual density matrix. Phys. Rev. Lett. 122(14), 141601 (2019). arXiv:1809.09109 [hep-th]

39. R.Q. Yang, C.Y. Zhang, W.M. Li, Holographic entanglement of purification for thermofield double states and thermal quench. JHEP 1901, 114 (2019). arXiv:1810.00420 [hep-th]

40. N. Bao, A. Chatwin-Davies, G.N. Remmen, Entanglement of purification and multiboundary wormhole geometries. JHEP 1902, 110 (2019). arXiv:1811.01983 [hep-th]

41. N. Bao, Minimal purifications, wormhole geometries, and the complexity=action proposal. arXiv:1811.03113 [hep-th]

42. N. Bao, G. Penington, J. Sorce, A.C. Wall, Beyond toy models: distilling tensor networks in full AdS/CFT. arXiv:1812.01171 [hepth]
43. P. Caputa, M. Miyaji, T. Takayanagi, K. Umemoto, Holographic entanglement of purification from conformal field theories. Phys. Rev. Lett. 122(11), 111601 (2019). arXiv:1812.05268 [hep-th]

44. W.Z. Guo, Entanglement of purification and projective measurement in CFT. Phys. Lett. B 797, 134934 (2019). arXiv:1901.00330 [hep-th]

45. P. Liu, Y. Ling, C. Niu, J.P. Wu, Entanglement of purification in holographic systems. JHEP 1909, 071 (2019). arXiv:1902.02243 [hep-th]

46. A. Bhattacharyya, A. Jahn, T. Takayanagi, K. Umemoto, Entanglement of purification in many body systems and symmetry breaking. Phys. Rev. Lett. 122(20), 201601 (2019). arXiv:1902.02369 [hepth]

47. M. Ghodrati, X.M. Kuang, B. Wang, C.Y. Zhang, Y.T. Zhou, The connection between holographic entanglement and complexity of purification. JHEP 1909, 009 (2019). arXiv:1902.02475 [hep-th]

48. K. Babaei Velni, M.R. Mohammadi Mozaffar, M.H. Vahidinia, Some Aspects of Holographic Entanglement of Purification. JHEP 1905, 200 (2019). arXiv: 1903.08490 [hep-th]

49. N. Jokela, A. Pönni, Notes on entanglement wedge cross sections. JHEP 1907, 087 (2019). [arXiv:1904.09582 [hep-th]]

50. W.Z. Guo, Entanglement of purification and disentanglement in CFTs. JHEP 1909, 080 (2019). [arXiv:1904.12124 [hep-th]]

51. S. Dutta, T. Faulkner, A canonical purification for the entanglement wedge cross-section. arXiv:1905.00577 [hep-th]

52. J. Kudler-Flam, M. Nozaki, S. Ryu, M.T. Tan, Quantum vs. classical information: operator negativity as a probe of scrambling. arXiv:1906.07639 [hep-th]

53. Y. Kusuki, K. Tamaoka, Dynamics of entanglement wedge cross section from conformal field theories. arXiv:1907.06646 [hep-th]

54. Y. Kusuki, J. Kudler-Flam, S. Ryu, Derivation of holographic negativity in $\mathrm{AdS}_{3} / \mathrm{CFT}_{2}$. Phys. Rev. Lett. 123(13), 131603 (2019). arXiv:1907.07824 [hep-th]

55. K. Umemoto, Quantum and classical correlations inside the entanglement wedge. arXiv:1907.12555 [hep-th]

56. H.S. Jeong, K.Y. Kim, M. Nishida, Reflected entropy and entanglement wedge cross section with the first order correction. arXiv: 1909.02806 [hep-th]

57. N. Bao, N. Cheng, Multipartite reflected entropy. JHEP 1910, 102 (2019). [arXiv:1909.03154 [hep-th]]

58. J. Levin, O. DeWolfe, G. Smith, Correlation measures and distillable entanglement in AdS/CFT. arXiv:1909.04727 [hep-th]

59. Y. Kusuki, K. Tamaoka, Entanglement wedge cross section from CFT: dynamics of local operator quench. arXiv:1909.06790 [hepth]

60. D. Marolf, CFT sewing as the dual of AdS cut-and-paste. arXiv: 1909.09330 [hep-th]

61. N. Cheng, Minimizations over geometric extensions in holography. arXiv:1909.09334 [hep-th]

62. J. Chu, R. Qi, Y. Zhou, Generalizations of reflected entropy and the holographic dual. arXiv:1909.10456 [hep-th]

63. C. Akers, P. Rath, Entanglement wedge cross sections require tripartite entanglement. arXiv:1911.07852 [hep-th]

64. K.B. Jaydeep, V. Malvimat, H. Parihar, B. Paul, G. Sengupta, On minimal entanglement wedge cross section for holographic entanglement negativity. arXiv:2002.10272 [hep-th]

65. J. Boruch, Entanglement wedge cross-section in shock wave geometries. arXiv:2006.10625 [hep-th]

66. I. Bakhmatov, N.S. Deger, J. Gutowski, Eó Colgáin, H. Yavartanoo, Calibrated entanglement entropy. JHEP 1707, 117 (2017). arXiv:1705.08319 [hep-th]

67. T. Hirata, T. Takayanagi, AdS/CFT and strong subadditivity of entanglement entropy. JHEP 0702, 042 (2007). [hep-th/0608213]

68. T. Nishioka, T. Takayanagi, AdS bubbles. Entropy and closed string tachyons. JHEP 0701, 090 (2007). hep-th/0611035 
69. I.R. Klebanov, D. Kutasov, A. Murugan, Entanglement as a probe of confinement. Nucl. Phys. B 796, 274 (2008). arXiv:0709.2140 [hep-th]

70. M. Headrick, Entanglement Renyi entropies in holographic theories. Phys. Rev. D 82, 126010 (2010). arXiv:1006.0047 [hep-th]

71. A. Frank, A.V. Karzanov, A. Sebo, On integer multiflow maximization. SIAM J. Discr. Math. 10, 158 (1997)

72. A. Schrijver, Combinatorial Optimization: Polyhedra and Efficiency, vol. 24 (Springer, Berlin, 2003)

73. N. Bao, S. Nezami, H. Ooguri, B. Stoica, J. Sully, M. Walter, The holographic entropy cone. JHEP 09, 130 (2015). arXiv:1505.07839 [hep-th]

74. N. Bao, M. Mezei, On the entropy cone for large regions at late times. arXiv:1811.00019 [hep-th]
75. S. Hernández Cuenca, Holographic entropy cone for five regions. Phys. Rev. D 100(2), 026004 (2019). arXiv:1903.09148 [hep-th]

76. B. Czech, X. Dong, Holographic entropy cone with time dependence in two dimensions. JHEP 10, 177 (2019). arXiv:1905.03787 [hep-th]

77. N. Bao, N. Cheng, S. Hernández-Cuenca, V.P. Su, the quantum entropy cone of hypergraphs. arXiv:2002.05317 [quant-ph]

78. M. Walter, F. Witteveen, Hypergraph min-cuts from quantum entropies. arXiv:2002.12397 [quant-ph]

79. N. Bao, N. Cheng, S. Hernández-Cuenca, V.P. Su, A gap between the hypergraph and stabilizer entropy cones. arXiv:2006.16292 [quant-ph] 